\title{
Netrin-1 overexpression in kidney proximal tubular epithelium ameliorates cisplatin nephrotoxicity
}

\author{
Amala Rajasundari ${ }^{1}$, Laurent Pays ${ }^{2}$, Patrick Mehlen $^{2}$ and Ganesan Ramesh ${ }^{1}$
}

Netrin-1, a multifunctional laminin-related protein is widely expressed in various tissues, including kidney. The pathophysiological roles of netrin-1 in toxic acute kidney injury are unknown. To determine the role of netrin-1 in cisplatin-induced nephrotoxicity, we used netrin-1 transgenic mice that overexpress netrin-1 in the proximal tubular epithelium using the fatty acid binding protein promoter. Administration of cisplatin caused severe renal injury in WT mice but not in netrin-1 transgenic mice. Functional improvement was associated with better preservation of morphology, reduced cytokine expression and oxidative stress in the kidney, and reduced serum and urine cytokine and chemokine levels of transgenic mice as compared with WT mice. Cisplatin induced an increase in neutrophil infiltration into the kidney of WT mice, which was not significantly reduced in netrin-1 transgenic mice. Interestingly, ischemia reperfusion induced a large increase in apoptosis in WT mice but not in netrin-1 transgenic mice (215 \pm 40 vs $94 \pm 20$ cells $/ 5$ HPF $(\times 400), P<0.0001)$, which was associated with reduced caspase- 3 and p53 activation in the transgenic kidney. These results suggest that netrin-1 protects renal tubular epithelial cells against cisplatin-induced kidney injury by suppressing apoptosis and inflammation.

Laboratory Investigation (2011) 91, 1717-1726; doi:10.1038/labinvest.2011.126; published online 29 August 2011

KEYWORDS: apoptosis; cell proliferation; ischemia reperfusion injury; netrin-1

Cisplatin (cis-diamminedichloroplatinum(II)) is an effective chemotherapeutic drug used in the treatment of many solid-organ cancers, including those of the head, neck, lung, testis, ovary, and breast. While toxicities include ototoxicity, gastrotoxicity, myelosuppression, and allergic reactions, ${ }^{1}$ the main dose-limiting, most common side effect of cisplatin is nephrotoxicity or acute kidney injury, which occurs in $20-30 \%$ of patients. ${ }^{2,3}$ Several recent studies have documented that cisplatin-induced acute kidney injury is an inflammatory disease associated with large increase in cell death by apoptosis. ${ }^{4-7}$ However, regulation of apoptosis and inflammation in cisplatin nephrotoxicity by endogenous molecules like netrin-1 is unknown.

Netrin-1, a diffusible laminin-related protein, is highly expressed outside the nervous system and its most abundant mRNA expression is found in the kidney. ${ }^{8,9}$ However, the function of netrin- 1 in tissues outside the nervous system is not clear. In recent studies using both in vitro and in vivo systems, netrin- 1 was shown to have a role in angiogenesis, ${ }^{10-12}$ cell migration, ${ }^{13}$ tissue morphogenesis, ${ }^{14,15}$ tumor progression and growth, ${ }^{16,17}$ and regulation of inflammation. ${ }^{9}$ Our most recent studies showed that administration of recombinant netrin-1 or transgenic overexpression in kidney prevented renal dysfunction by suppressing apoptosis and inflammation. ${ }^{8}$ However, whether netrin-1 has any protective role in toxic acute kidney injury is unknown.

To determine the role of netrin-1 in cisplatin-induced acute kidney injury, we used mice that overexpress netrin-1 in the proximal tubular epithelial cells using the liver fatty acid binding protein promoter. ${ }^{18}$ We characterized netrin-1 transgene expression in the kidney in our earlier study. ${ }^{19}$ These transgenic mice showed normal kidney function and reproductive phenotype under basal conditions. Quantification of transgene expression by western blot showed that expression levels were more than five-fold greater as compared with wild-type mice. We subjected netrin-1 transgenic mice to cisplatin treatment and assessed kidney function. Our results show that netrin-1 transgenic mice are resistant to cisplatininduced kidney injury. This resistance was associated with reduced cytokine production, apoptosis, and p53 activation.

\footnotetext{
${ }^{1}$ Department of Medicine and Vascular Biology Center, Georgia Health Sciences University, Augusta, GA, USA and ${ }^{2}$ Apoptosis, Cancer and Development Laboratory, Equipe labellisée 'La Ligue', CNRS UMR, Centre Leon Berard, Lyon, France

Correspondence: Professor G Ramesh, PhD, Department of Medicine and Vascular Biology Center, CB-3330, Georgia Health Sciences University, 1459 Laney-Walker Boulevard, Augusta, GA 30912, USA.

E-mail: gramesh@georgiahealth.edu

Received 11 March 2011; revised 4 May 2011; accepted 23 May 2011
} 


\section{MATERIALS AND METHODS}

\section{Animals and Drug Administration}

Experiments were performed using 8-10-week-old male C57BL/6 mice and netrin-1 transgenic mice that overexpress netrin-1 in kidney under control of the fatty acid binding protein promoter. ${ }^{18,19}$ Netrin-1 transgenic mice were in the C57BL/6 background and were backcrossed for $>30$ generations. Transgenic mice were regularly bred with $\mathrm{C} 57 \mathrm{BL} / 6$ mice from Jackson Laboratory and either littermates or C57BL/6 WT mice from Jackson Laboratory weighing $\sim 25-30 \mathrm{~g}$ were used as a control. Mice were maintained on a standard diet, and water was freely available. This study was approved by IACUC at the Georgia Health Sciences University. Cisplatin was dissolved in saline at a concentration of $1 \mathrm{mg} / \mathrm{ml}$. Mice were given a single intraperitoneal injection of either saline or cisplatin $(20 \mathrm{mg} / \mathrm{kg}$ body weight). Animals were killed $72 \mathrm{~h}$ after cisplatin injection, and blood and kidney tissues were collected. Kidney tissues were processed for histology, TUNEL assay, neutrophil and p53 staining, and RNA isolation.

\section{Cell Culture}

Immortalized mouse proximal tubule cells, TKPTS $^{20}$ cells, kindly provided by Dr Bell-Reuss and Dr J Megyesi, were cultured in DMEM/F12 supplemented with glutamine, 7.5\% FBS and antibiotics, and were grown to confluence and maintained at $37^{\circ} \mathrm{C}$ in $5 \% \mathrm{CO}_{2}$. Cells were treated with cisplatin with/without netrin-1 for $24 \mathrm{~h}$ and harvested for RNA isolation at the end of the incubation.

\section{Renal Function}

Renal function was assessed by measurements of blood urea nitrogen (VITROS DT60II Chemistry slides, Ortho-clinical Diagnostics) and serum creatinine (cat no: DZ072B, Diazyme Labs, USA).

\section{Quantitation of mRNA by Real-time RT-PCR}

Real-time RT-PCR was performed in an Applied Biosystems Inc. 7700 Sequence Detection System (Foster City, CA, USA). In all, $1.5 \mu \mathrm{g}$ total RNA was reverse transcribed in a reaction volume of $20 \mu \mathrm{l}$ using Omniscript RT kit and random primers. The product was diluted to a volume of $150 \mu \mathrm{l}$, and $6 \mu \mathrm{l}$ aliquots were used as templates for amplification using the SYBR Green PCR amplification reagent (Qiagen) and gene-specific primers. The primer sets used were mouse TNF $\alpha$ (forward: GCATGATCCGCGACGTGGAA; reverse: AGATCCATGCCGTTG GCCAG), MCP-1 (forward: ATGCA GGTCCCTGTCATG; reverse: GCTTGAGGTGGTTGTGGA), ICAM-1 (forward: AGATCACATTCACGGTGCTG; reverse: CTTCAGAGGCAGGAAACAGG), netrin-1 (forward: AAGC CTATCACCCACCGGAAG; reverse: GCGCCACAGGAATCT TGATGC), osteopontin (forward: TCACCATTCGGATGAGT CTG-3'; reverse: ACTTGTGGCTCTGATGTTCC), hemeoxygenase-1 (HO-1) (forward: AGCATGCCCCAGGATTTG; reverse: AGCTCAATGTTGAGCAGGA), and TGF- $\beta 1$ (forward:
TGACGTCACTGGAGTTGTACGG; reverse: GGTTCATGTC ATGGATGGTGC). The amount of DNA was normalized to the $\beta$-actin signal amplified in a separate reaction (forward primer: AGAGGGAAATCGTGCGTGAC; reverse: CAATAGT GATGACCTGGCCGT).

\section{Serum Cytokine Measurement}

Serum cytokines and chemokines were measured using ELISA array kit from SA biosciences and ELISA kit from eBiosciences.

\section{Immunohistochemical Localization of p53 and Caspase-3}

Immunohistochemical localization of p53 and caspase- 3 was performed as described before. ${ }^{19}$ Briefly, paraffin-embedded sections were deparaffinized and hydrated. Endogenous peroxidase activity was blocked with $90 \%$ methanol $/ 3 \% \mathrm{H}_{2} \mathrm{O}_{2}$ followed by permeabilization with $0.2 \%$ Triton X-100 in PBS. Sections were then washed and blocked with PBS containing $5 \%$ donkey serum and $1 \%$ BSA. Primary antibodies included a goat anti-caspase-3 and rabbit anti-p53 (Cell Signaling Technologies, Inc.). Primary antibodies were detected using a biotin-conjugated secondary antibody followed by $\mathrm{ABC}$ reagent. Color was developed using metal-enhanced $D A B$ substrate. Peroxidase staining was visualized and photographed using an Olympus inverted microscope with a CCD camera attached to it.

\section{TACS TdT In Situ Apoptosis Detection}

To identify apoptotic cells, tissue sections were stained using TACS TdT in situ Apoptosis Detection kit (R\&D Systems, Inc.) according to the manufacturer's instruction. Briefly, tissue sections were deparaffinized, hydrated, and washed with PBS. Sections were digested with proteinase $\mathrm{K}$ for $15 \mathrm{~min}$ at $24^{\circ} \mathrm{C}$. Slides were then washed and endogenous peroxidase activity was quenched with $3 \% \mathrm{H}_{2} \mathrm{O}_{2}$ in methanol. Slides were washed and incubated with TdT labeling reaction mix at $37^{\circ} \mathrm{C}$ for $1 \mathrm{~h}$ and then with streptavidin-HRP. Color was developed using TACS blue label substrate solution. Slides were washed, counterstained, and mounted with Permount. Sections were photographed, and labeled cells were counted and quantified.

\section{Histology}

Kidney tissue was fixed in buffered $10 \%$ formalin for $12 \mathrm{~h}$ and then embedded in paraffin wax. For assessment of injury, $5 \mu \mathrm{M}$ sections were stained with periodic acid-Schiff (PAS). Acute tubular necrosis was assessed in the outer strip of the outer medulla and cortex using a semiquantitative scale in which the percentage of tubules showing epithelial necrosis, brush-border loss and cast formation was assigned a score: $0=$ normal, $1=<10 \%, 2=11-25 \%, 3=26-45 \%$, $4=46-75 \%$, and $5=>76 \% .^{21,22}$ Ten fields of $\times 40$ magnification were examined and averaged. The slides were scored in a blinded manner and de-identified. To quantify leukocyte 
infiltration, sections were stained with rat anti-mouse neutrophils antibody (SEROTECH) (1:200 dilution) followed by goat anti-rat biotin conjugate. Color was developed after incubation with $\mathrm{ABC}$ reagent (Vector Lab). Stained sections were photographed and five $\times 40$ fields of neutrophils were examined for quantitation of leukocytes.

\section{Statistical Methods}

All assays were performed in duplicate. The data are reported as mean \pm s.e.m. Statistical significance was assessed by an unpaired, two-tailed Student's $t$-test for single comparison or ANOVA for multiple comparisons.

\section{RESULTS}

\section{Netrin-1 Transgenic Mice Are Resistant to Cisplatin-Induced Renal Dysfunction}

The role of netrin-1 in toxic acute kidney injury is unknown. To determine the role of netrin-1 in cisplatin-induced kidney injury, WT and netrin-1 transgenic mice were administered with cisplatin or saline. Renal function was determined by measuring serum creatinine. As shown in Figure 1a, WT mice developed severe renal dysfunction. Serum creatinine was increased significantly by $24 \mathrm{~h}$ and continued to climb up to $72 \mathrm{~h}$. However, netrin-1 transgenic mice displayed lower serum creatinine levels at all time points. Saline-treated WT and netrin-1 transgenic mice did not develop any renal dysfunction. Improved renal function in the transgenic mice was associated with improved tissue morphology as determined by reduction in necrosis score as compared with WT mice (not shown). Semiquantitative scoring of tubule injury was significantly higher in WT kidneys $(3.8 \pm 0.1)$ than in kidneys from netrin- 1 transgenic mice $(2.4 \pm 0.6)$ at $72 \mathrm{~h}$ after cisplatin administration $(P<0.05$, $n=4-6$ per genotype).

\section{Netrin-1 Overexpression Reduced Cytokine, Adhesion Molecule, and Oxidative Stress Marker Expression}

Several in vitro and in vivo studies suggested that cisplatin nephrotoxicity is an inflammatory disease characterized by exacerbated inflammatory response from the kidney. ${ }^{7,23}$ Moreover, netrin-1 administration reduced inflammation and inhibited leukocyte migration. ${ }^{8,9}$ To determine whether netrin-1 overexpression reduces inflammation, expression of several cytokines, chemokines, and adhesion molecules was determined by real-time RT-PCR. Cisplatin administration induced a significant increase in MCP-1, TNF $\alpha$, RANTES, ICAM-1, complement C3, osteopontin, and HO-1 (Figure 1b). Overexpression of netrin-1 significantly reduced RANTES, complement C3, ICAM-1, osteopontin, and HO-1 mRNA levels.

To determine if suppression of mRNA expression correlates with protein levels, cytokines and chemokines in serum and urine were quantified by ELISA. Administration of cisplatin increased serum levels of several cytokines (IL-6 and IL-10) and chemokines (MCP-1, MIG, eotaxin, and KC) that
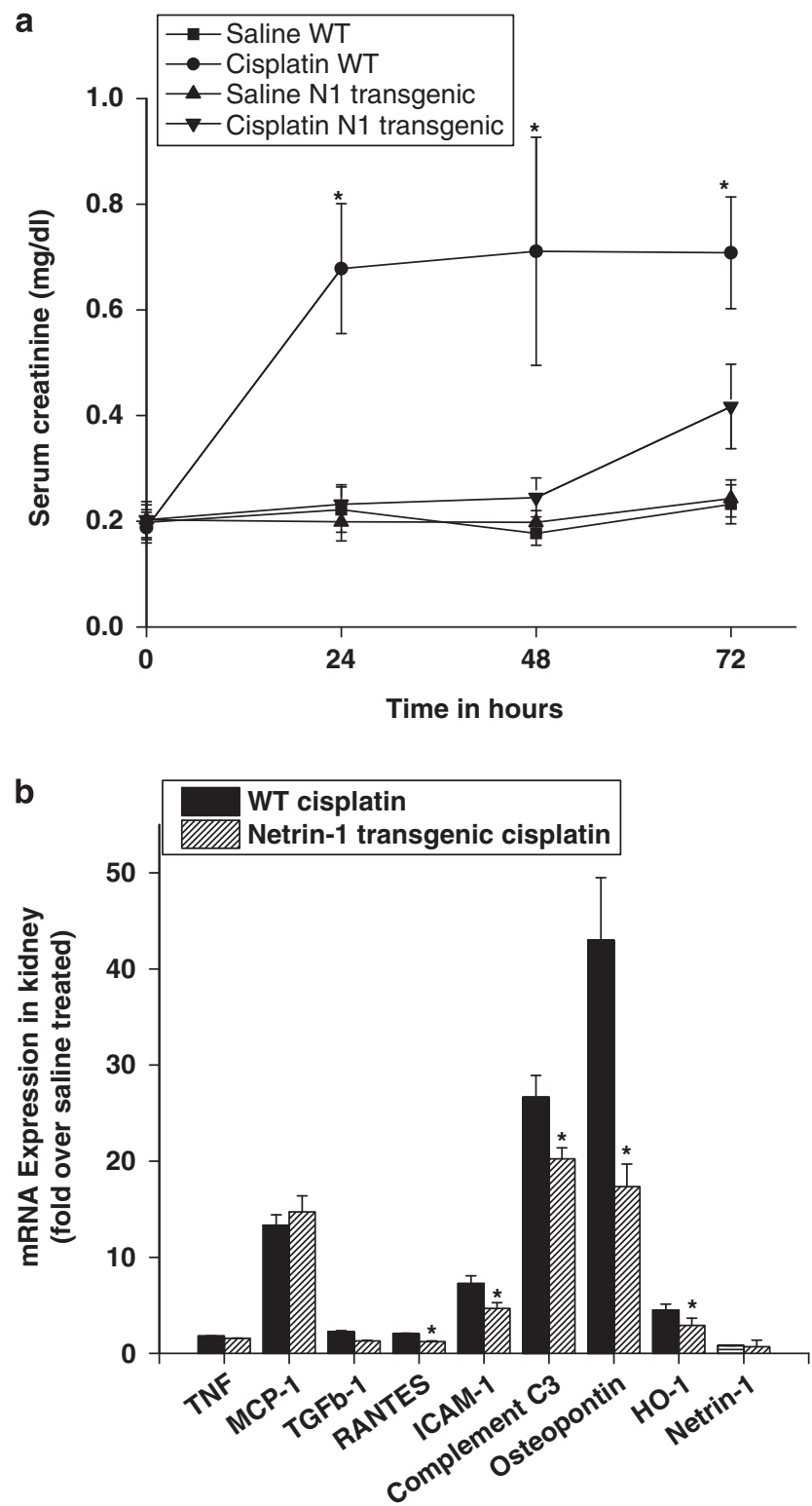

Figure 1 Effect of overexpression of netrin-1 on cisplatin-induced renal dysfunction. Serum creatinine (a) was measured at different times after cisplatin or saline administration in WT and netrin-1 transgenic mice. Values are means \pm s.e.m. ${ }^{*} P<0.01$ vs all other groups. Values are mean \pm s.e.m.; $n=8-10$ for each group. Cytokine and chemokine, adhesion molecule, and complement expression were quantified by real-time RT-PCR (b) and expressed as fold over saline-treated animals. ${ }^{\star} P<0.05$ vs WT cisplatin-treated mice.

were significantly reduced in netrin- 1 transgenic mice treated with cisplatin at $24 \mathrm{~h}$ (Figure 2). Interestingly, IL-4 and IL-13 levels in serum are elevated in netrin-1 transgenic animals but not WT mice treated with cisplatin. A more dramatic increase of cytokine and chemokine levels was seen in urine of WT mice. As shown in Figure 3, cytokine (IL-2, IL-4, IL-5, IL-6, IL-10, IL-12, IL-13, IL-17, IL-23, IFN $\gamma$, TNF $\alpha$, and TGF $\beta 1$ ) and chemokine (RANTES, MCP- 1, MIP- $1 \alpha$, MIP- $1 \beta$, 

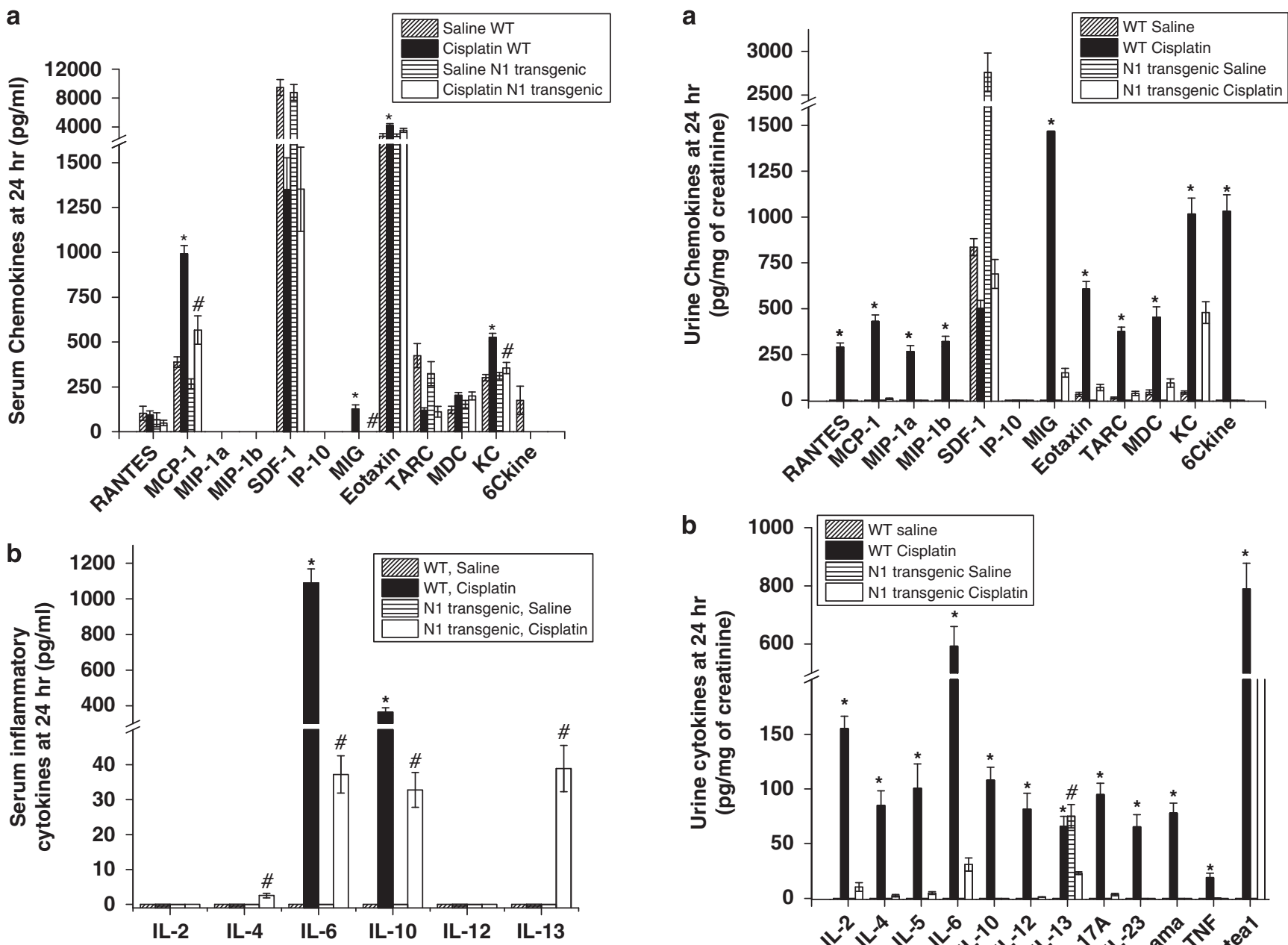

Figure 2 Effect of netrin-1 overexpression on serum cytokine (b) and chemokine levels (a). Cytokine and chemokine levels in serum were measured $24 \mathrm{~h}$ after cisplatin or saline administration in WT and netrin-1 transgenic animals. Netrin-1 overexpression suppressed cisplatin-induced cytokine and chemokine increase in serum. Values are means \pm s.e.m. ${ }^{*} P<0.05$ vs WT saline-treated mice. ${ }^{\#} P<0.05$ vs WT cisplatin-treated mice; $n=6$.

MIG, eotaxin, TARC, MDC, KC, and 6Ckine) levels were significantly elevated in WT mice at $24 \mathrm{~h}$ after cisplatin administration. All these cytokine and chemokine levels were suppressed in netrin-1 transgenic mice except IL-4 and IL-13 which elevated in both basal and cisplatin-treated netrin-1 transgenic mice. Changes in cytokine and chemokine levels in serum are moderate as compared with urine levels which showed a dramatic increase, suggesting that renal tubular epithelial cells may be a major source of these cytokines and chemokines. These results suggest that netrin-1 is a major regulator of cytokine and chemokine expression in kidney.

To determine whether urinary cytokines were derived from tubular epithelial cells after cisplatin administration, mouse proximal tubular epithelial cells were treated with $25 \mu \mathrm{M}$ cisplatin and with/without netrin-1 for $24 \mathrm{~h}$. Expression of $\mathrm{TNF} \alpha$ was quantified by real-time RT-PCR as described in

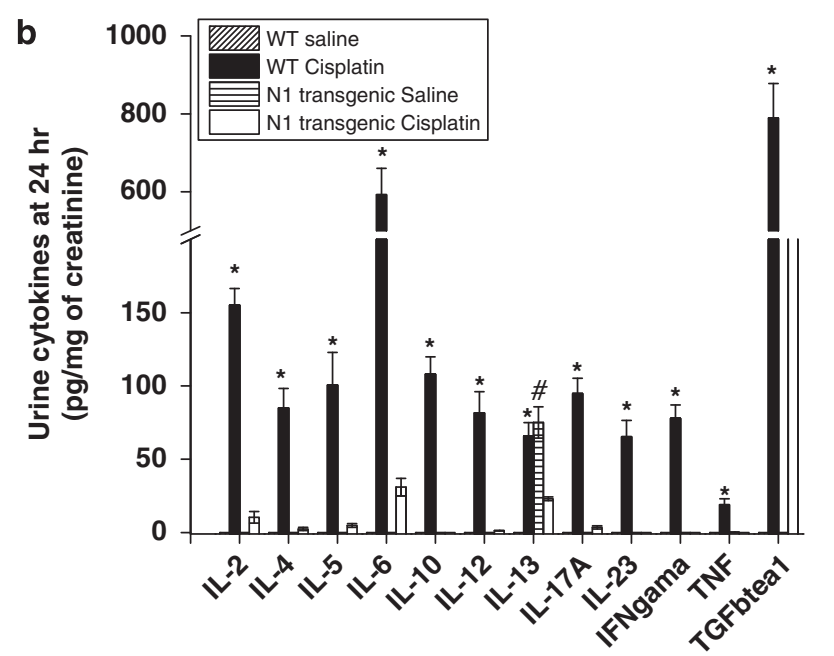

C
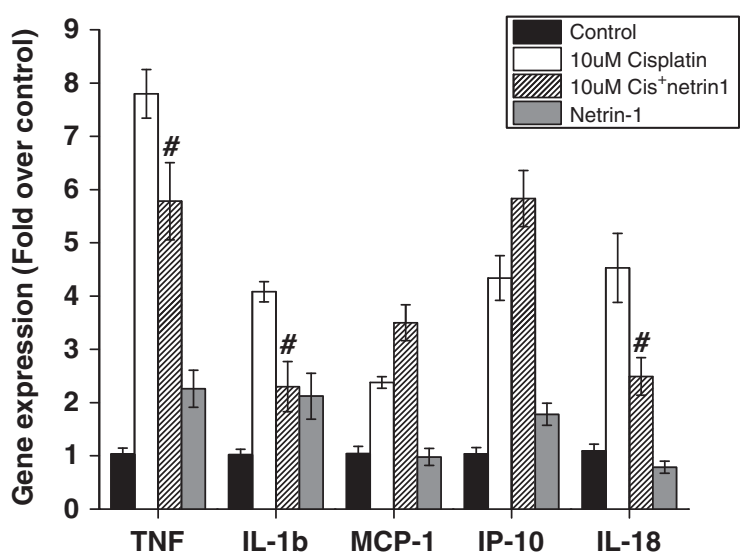

Figure 3 Effect of netrin-1 overexpression on urine cytokine and chemokine levels. Chemokine (a) and cytokine (b) levels in urine were measured $24 \mathrm{~h}$ after cisplatin or saline administration in WT and netrin-1 transgenic animals. Netrin-1 overexpression suppressed cisplatin-induced cytokine and chemokine increase in urine. Values are means \pm s.e.m. ${ }^{*} P<0.001$ vs all other groups; $n=6$. Netrin-1 suppresses cisplatin-induced cytokine expression in proximal tubular epithelial cells in vitro (c). ${ }^{\#} P<0.05$ vs cisplatin treated. $n=4$. 
Materials and Methods. As shown in Figure 3c, cisplatin treatment increased TNF $\alpha$ expression, which is significantly reduced in netrin-1-treated cells, suggesting that netrin-1 regulates cytokine and chemokine production in response to cisplatin in proximal tubular epithelial cells.
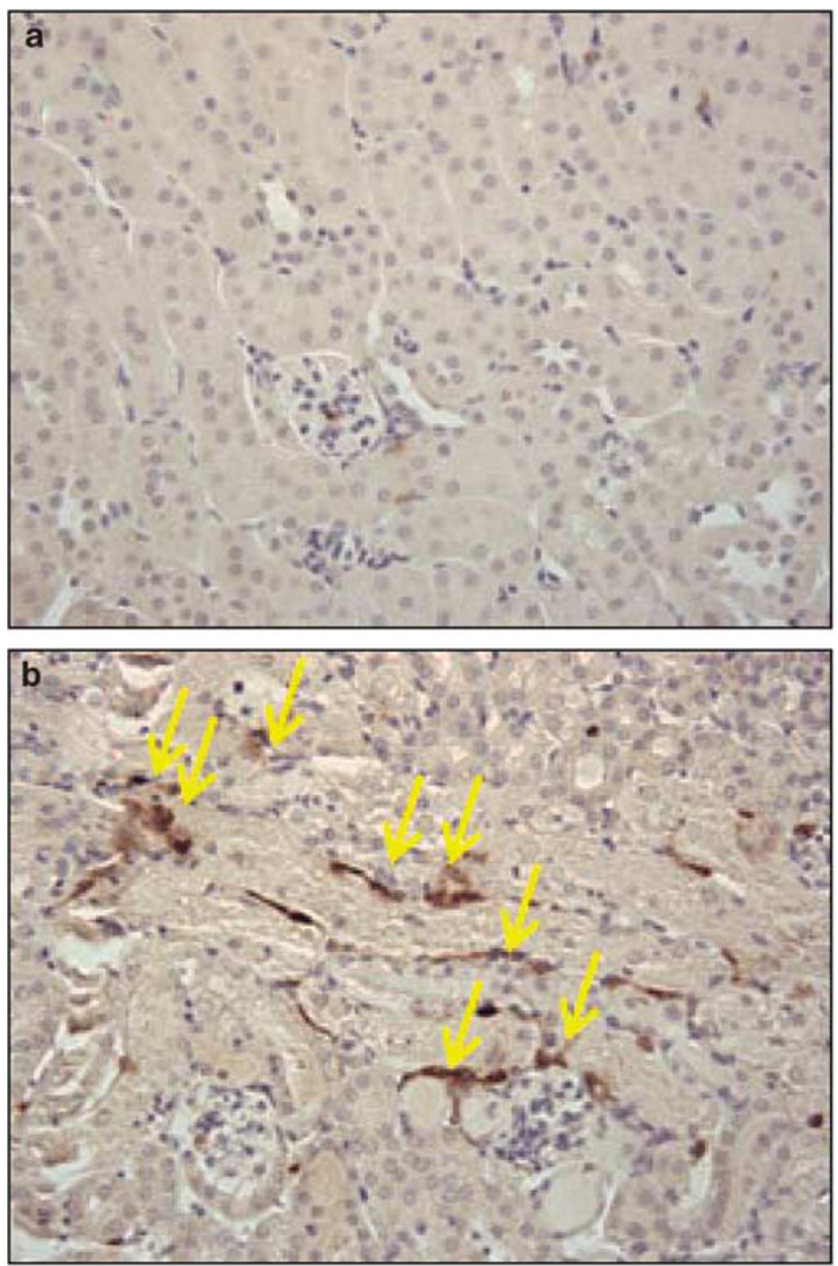

e

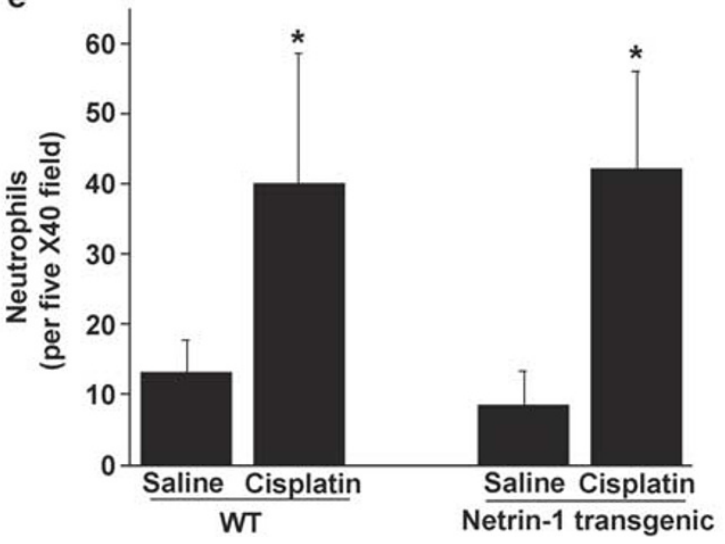

\section{Netrin-1 Overexpression in Tubular Epithelial Cells Does Not Reduce Neutrophil Infiltration}

Earlier studies suggested that intravenous administration of recombinant netrin-1 reduced neutrophil infiltration into the kidney after ischemia reperfusion ${ }^{8}$ and reduced leukocyte
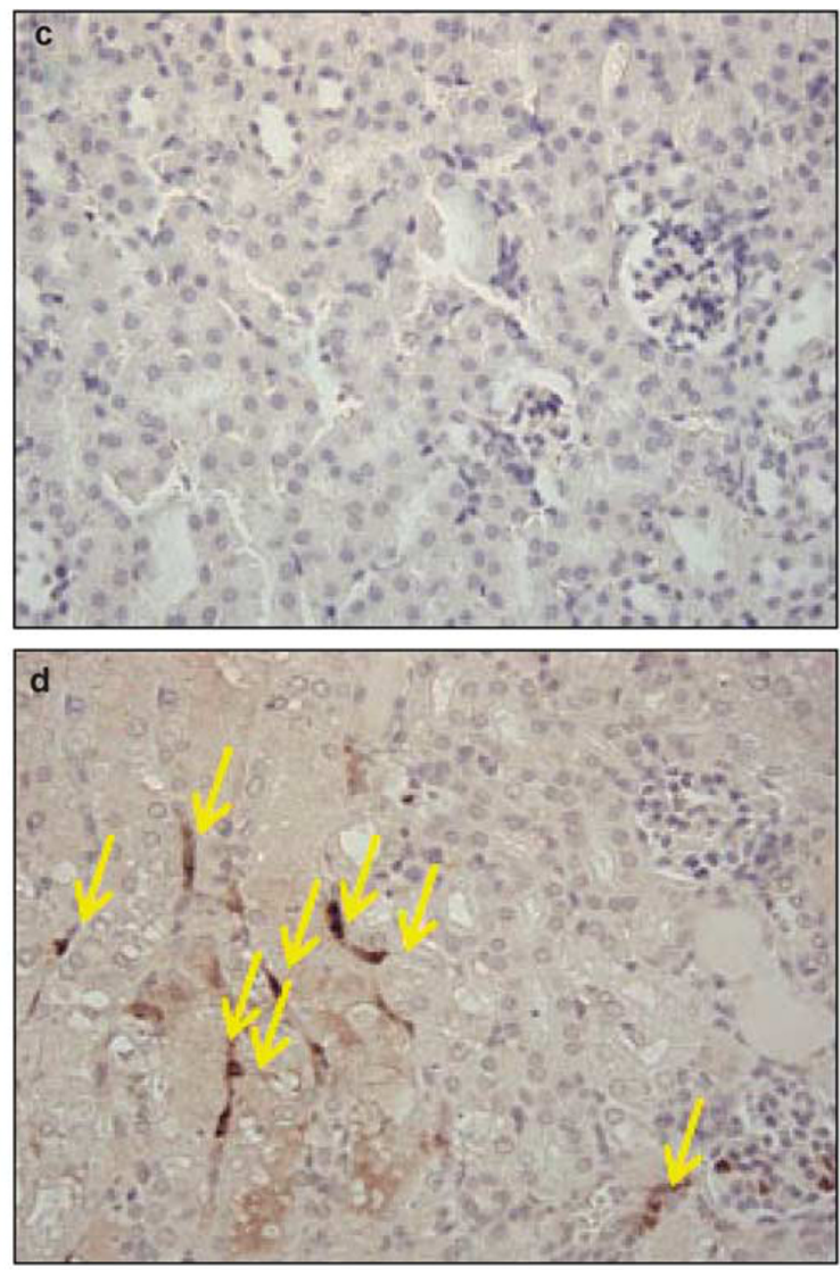

Figure 4 Effect of netrin-1 overexpression on neutrophil infiltration. Sections were fixed and stained for neutrophils as described in Material and Methods. (a) Saline-treated WT mice. (b) Netrin-1 transgenic mice showed few neutrophils. Seventy-two hours after cisplatin administration, both WT (c) and netrin-1 transgenic mice kidney (d) had shown increased neutrophil staining. (e) Quantitative summary data of neutrophil infiltration $\left({ }^{*} P<0.001\right.$ vs saline treated). $n=4-6$. 
migration during sepsis ${ }^{9}$ but not when netrin-1 was overexpressed in proximal tubule in an ischemia reperfusion injury model. To determine whether the observed protective effect of tubule-specific overexpression of netrin-1 is mediated, in part, through reducing leukocyte infiltration, tissue sections were stained with an anti-neutrophil antibody (Figure 4). Saline-treated WT and transgenic mouse kidneys had very few neutrophils (Figure $4 \mathrm{a}$ and b). Cisplatin administration
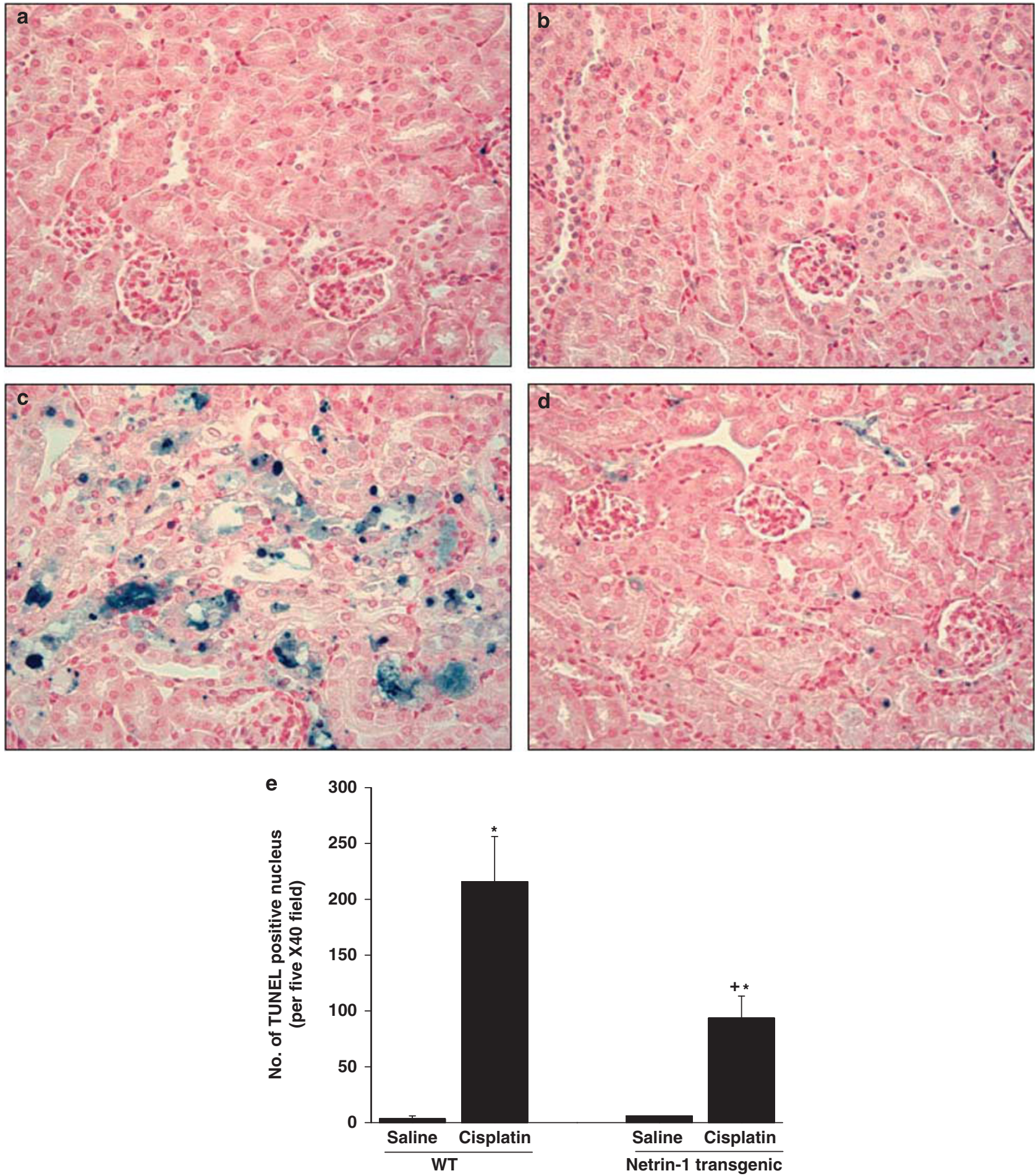

Figure 5 Effect of netrin-1 overexpression on cisplatin-induced apoptosis. Apoptotic cells were identified using TUNEL staining (a-d). Sham-operated WT and transgenic mouse kidney did not show any apoptotic cells $(\mathbf{a}, \mathbf{b})$. Seventy-two hours after cisplatin administration, the number of apoptotic cells (blue staining) was increased dramatically in WT (c) whereas only a few cells were positive for apoptosis in transgenic animals (d). (e) Quantification of apoptotic cells. Apoptotic-positive cells were counted in five random $\times 40$ fields. ${ }^{\star} P<0.001$ vs saline treated. ${ }^{+} P<0.001$ vs WT cisplatin treated; $n=4-6$. 
dramatically increased neutrophil infiltration in WT (Figure $4 c)$ and in netrin- 1 transgenic mice (Figure $4 \mathrm{~d}$ ). These results suggest that the observed protective effect of netrin-1 overexpression is not due to suppression of neutrophil infiltration.
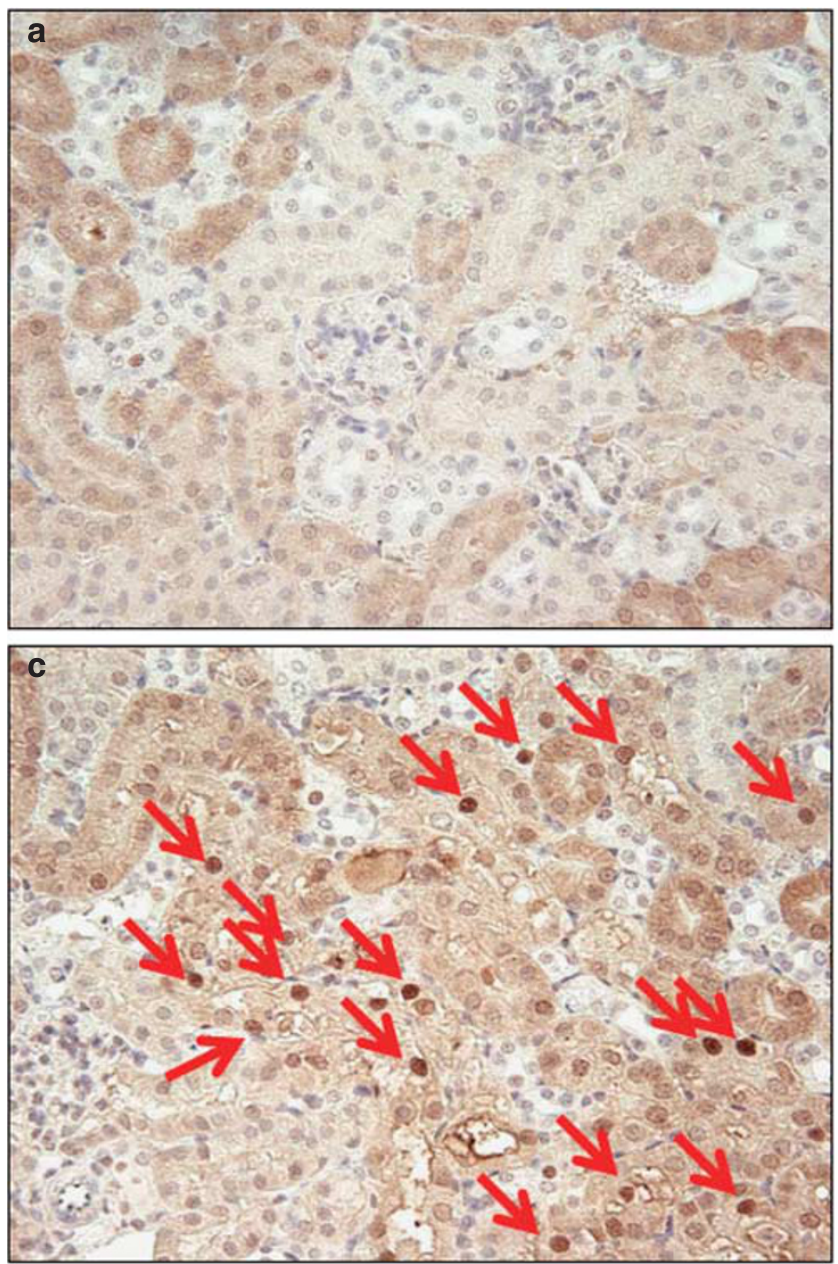

\section{Netrin-1 Overexpression Reduces Cisplatin-Induced Tubular Epithelial Cell Apoptosis}

Netrin-1 was shown to be a survival factor for intestinal epithelial cells and, in the absence of netrin-1, the receptors
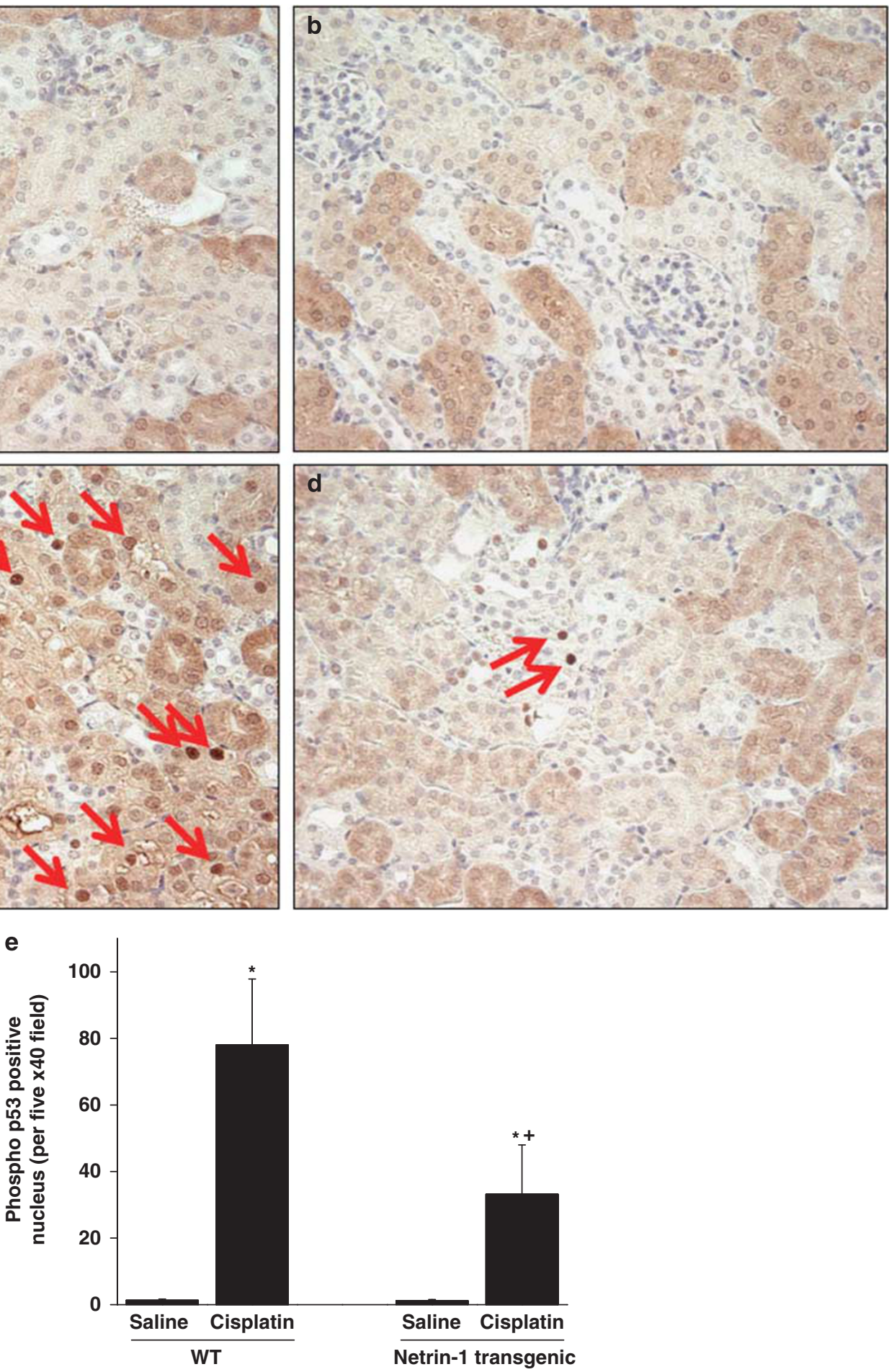

Figure 6 Effect of netrin-1 overexpression on cisplatin-induced activation of p53. Phospho p53 was immunolocalized in formalin-fixed sections as described in Materials and Methods (a-d). Saline-treated WT and transgenic mouse kidney did not show any p53-positive nuclei (a, b). Seventy-two hours after cisplatin administration, the number of p53-positive cells (arrows) was increased dramatically in WT (c) whereas only a few cells were positive for p53 in transgenic mice kidney (d). (e) Quantitation of p53-positive nuclei. Phospho p53-positive nuclei were counted in five random $\times 40$ fields. ${ }^{\star} P<0.005$ vs saline treated. ${ }^{+} P<0.001$ vs WT cisplatin treated; $n=4-6$. 
DCC and UNC5B are known to induce apoptosis. ${ }^{18,24,25}$ Cisplatin administration is known to induce apoptosis in tubular epithelial cells. In that context, inhibition of apoptosis leads to improved renal function. ${ }^{5,26}$ To determine whether netrin-1 alters cisplatin-induced apoptosis, apoptotic cells were quantified using TACS TdT staining. Salinetreated WT (Figure 5a) and netrin-1 transgenic (Figure 5b) kidneys did not show any apoptotic cells. Administration of cisplatin induced a large increase in apoptosis in WT mice by $72 \mathrm{~h}$ (Figure 5c). However, cisplatin-induced apoptosis was drastically reduced in netrin-1 transgenic kidneys (Figure 5d). Quantification of apoptotic cells showed a significant decrease in transgenic mice as compared with WT mice treated with cisplatin (Figure 5e).

To determine the pathways through which apoptosis is suppressed, tissue sections were also stained with antibodies against phospho p53 and active caspase-3. As shown in Figure 6 , cisplatin induced a large increase in phospho p53 staining in WT mice (Figure 6c), which is drastically and significantly reduced in netrin-1 transgenic mice (Figure 6d). Quantitative data are provided in Figure 6e, which also showed a significant decrease in number of p53-positive nucleus in netrin-1 transgenic mouse kidney as compared with WT mouse kidney. Activation of $\mathrm{p} 53$ by cisplatin leads to the activation of caspases, and netrin-1 is known to suppress caspase activation. Therefore, we determined whether netrin-1 suppresses capase3 activation. As shown in Figure 7, caspase-3 staining in saline-treated WT (Figure 7a) and netrin-1 transgenic mouse kidney (Figure $7 \mathrm{~b}$ ) is not increased. However, administration of cisplatin increased active caspase- 3 staining in WT mouse kidney, which is suppressed in netrin-1 transgenic mice kidney. These data suggest that suppression of p53 pathwaymediated activation of caspase may contribute to netrin-1mediated protection of kidney against cisplatin injury.

\section{DISCUSSION}

The present study provides evidence that netrin-1, a lamininrelated neuronal guidance cue, when expressed in proximal tubule cells, can prevent renal dysfunction and epithelial cell apoptosis in response to cisplatin administration.
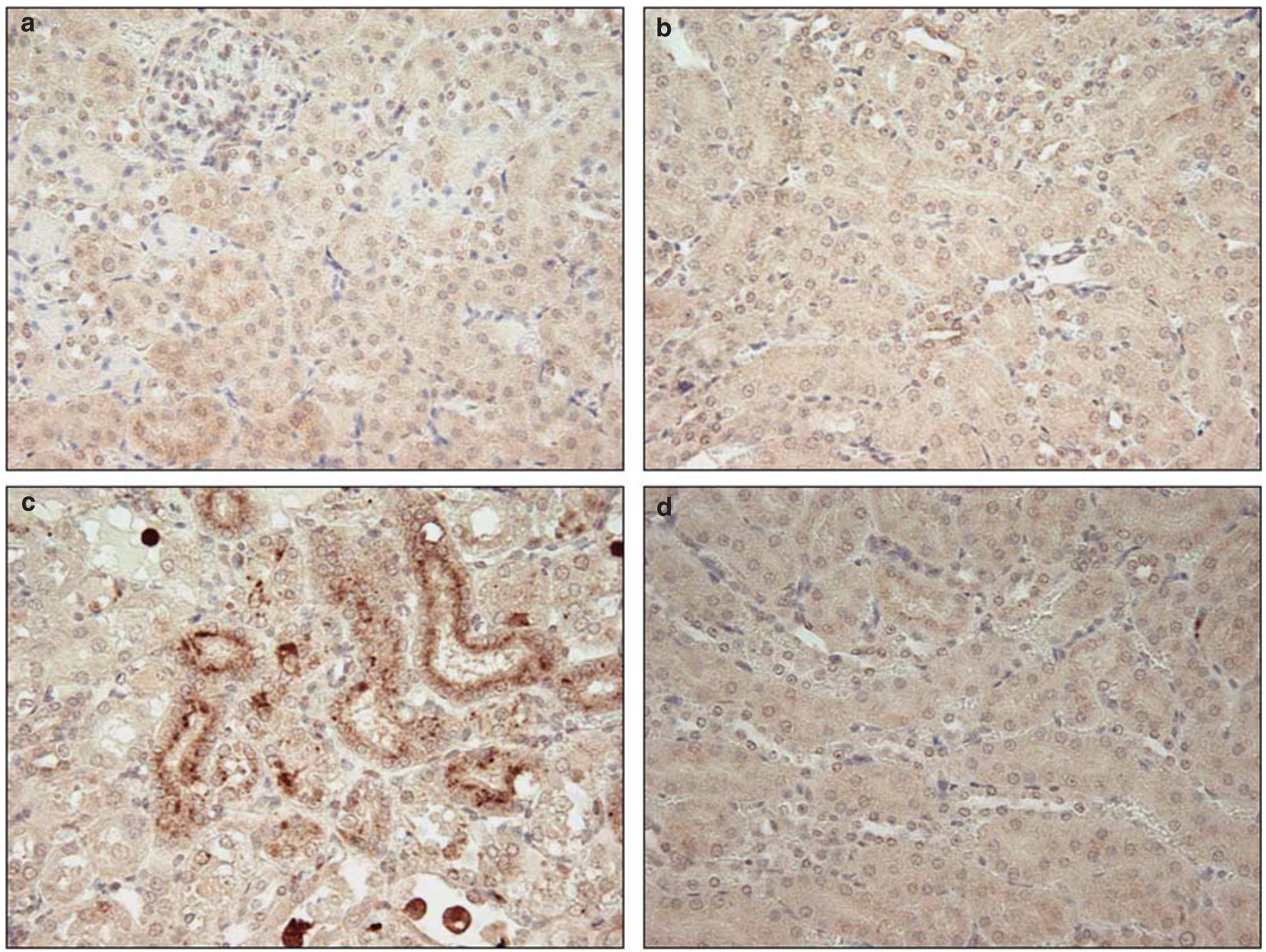

Figure 7 Effect of netrin-1 overexpression on cisplatin-induced caspase-3 activation. Kidneys from saline-treated WT and transgenic mice did not show any staining for active caspase-3 $(\mathbf{a}, \mathbf{b})$. Seventy-two hours after cisplatin administration, tubular epithelial cells positive for active caspase-3 were increased in WT mice (c) but not in netrin-1 transgenic mice (d). 
Furthermore, netrin- 1 suppressed caspase- 3 and p53 activation in tubular epithelial cells and reduced inflammatory cytokine expression and excretion. These results indicate that netrin-1 has a key role in toxic acute kidney injury by suppressing apoptosis and inflammatory cytokine expression.

Our earlier studies had determined that netrin-1 transgenic mice had normal kidney function and exhibit histological feature under basal conditions, ${ }^{19}$ suggesting that overexpression of netrin-1 in tubular epithelial cells is not altering normal kidney function. Our earlier studies showed that administration of cisplatin to mice rapidly induced netrin- 1 in the renal tubular epithelial cells and excreted in urine. ${ }^{27}$ However, the role of netrin-1 in cisplatin-induced kidney is unknown. The present study determined that netrin-1 overexpression protected against cisplatin-induced acute kidney injury. This effect is likely due to the marked suppression of apoptosis in the transgenic mice. Although several in vitro and in vivo studies in other tissues have demonstrated a role for netrin-1 in inhibiting apoptosis, ${ }^{17,18,28-30}$ this is the first study to show the anti-apoptotic role of netrin-1 in kidney tubules in a model of toxic acute kidney injury.

Netrin-1 receptors (DCC and UNC5) are also called dependence receptors as these receptors are known to induce apoptosis when they are not bound to netrin- $1 .{ }^{24}$ But the presence of netrin-1 UNC5b mediates cell survival by activating survival pathways such as like PI3K and ERK pathways. Moreover, netrin-1 is known to suppress caspase and p53 activation leading to suppression of apoptosis. ${ }^{29,31,32}$ Along this line, netrin- 1 administration prevented p53 activation and ischemia-induced apoptosis in brain. ${ }^{30}$ Both caspase and p53 are activated by cisplatin in kidney and are known to mediate kidney injury. ${ }^{5,33}$ Since netrin-1 is known to suppress p53 and caspase activation in other tissues, ${ }^{30,32,34}$ it suggests that a similar mechanism may exist in kidney as well. Our results support these findings that netrin-1-mediated protection against cisplatin-induced kidney injury is in part through suppression of caspase- 3 and p53 activation (Figures 6 and 7).

Inflammatory mechanisms have a key role in cisplatininduced kidney injury. ${ }^{6,7,23}$ Recent studies suggest that netrin-1 limits inflammation by inhibiting leukocyte migration via UNC5B receptors. ${ }^{9}$ Although intravenous administration of netrin-1 reduced ischemia reperfusion-induced neutrophil infiltration, tubule-specific overexpression in the present study did not influence neutrophil infiltration. This observation suggests that the observed protective effects in netrin- 1 transgenic mice against cisplatin-induced kidney injury are not through suppression of neutrophil infiltration. It is possible that the netrin-1 expressed in proximal tubule cells may not be available to the vascular compartment to act on the leukocytes. However, netrin-1 transgenic mice did suppress the expression and excretion of several pro-inflammatory chemokines and cytokines that are known to be involved in kidney injury by acting directly on the tubular epithelial cells. Therefore, some of the protection afforded by netrin-1 could be referable to this reduction in cytokine expression. The cell types that produce cytokines and chemokines are not clear in our study. However, earlier studies ${ }^{22,35}$ showed that renal epithelial cells are capable of producing several inflammatory cytokines and chemokines in response to cisplatin treatment.

Tubular epithelial cell-specific expression of netrin-1 suppressed a wide array of cytokine and chemokine expression except the Th2 cytokines IL-4 and IL-13 which were upregulated at $72 \mathrm{~h}$ after cisplatin treatment (data not shown). In contrast, Th2 cytokine expression is suppressed by netrin-1 in T cells. ${ }^{36}$ Whether netrin-1 regulates IL-4/IL-13 expression in renal epithelial cells is unknown. IL-13 is known to suppress tubular epithelial cell apoptosis and reduce sepsis and ischemic kidney injury. ${ }^{37-39}$ However, whether netrin-1 mediates part of its protective effect through IL-4 and IL-13 is unknown. Th2 cytokine is also known to exert an antiinflammatory phenotype by inducing macrophage polarization to M2 phenotype in addition to suppression of apoptosis in epithelial cells. The connection between IL-4/IL-13 and netrin-1 in renal epithelial cell functions needs to be explored further.

In summary, our findings support the view that netrin-1 is a strong pro-survival factor for proximal tubule cells and can suppress cisplatin-induced apoptosis possibly by suppressing p53 and caspase- 3 activation. Netrin-1-mediated suppression of inflammatory cytokine expression and positive regulation of Th2 cytokines suggests that part of the netrin- 1 effect on the kidney is mediated through IL-13. Our result suggests that netrin-1 may be a useful therapeutic molecule for treating cisplatin nephrotoxicity and other inflammatory diseases as well. The molecular mechanism by which netrin- 1 and Th2 cytokine interacts warrants further investigation.

\section{ACKNOWLEDGEMENTS}

This work was supported by grants to Ganesan Ramesh from American Society of Nephrology (Carl S. Gottschalk Research Scholar Grant) and R01 grant (5R01DK083379 - 02) from NIH-NIDDK.

\section{DISCLOSURE/CONFLICT OF INTEREST}

The authors declare no conflict of interest.

1. Hartmann Jr T, Lipp HP. Toxicity of platinum compounds. Expert Opin Pharmacother 2003;4:889-901.

2. Sastry J, Kellie SJ. Severe neurotoxicity, ototoxicity and nephrotoxicity following high-dose cisplatin and amifostine. Pediatr Hematol Oncol 2005;22:441-445.

3. Arany I, Safirstein RL. Cisplatin nephrotoxicity. Semin Nephrol 2003; 23:460-464.

4. Lau AH. Apoptosis induced by cisplatin nephrotoxic injury. Kidney Int 1999;56:1295-1298.

5. Pabla N, Dong Z. Cisplatin nephrotoxicity: mechanisms and renoprotective strategies. Kidney Int 2008;73:994-1007.

6. Zhang B, Ramesh G, Uematsu S, et al. TLR4 signaling mediates inflammation and tissue injury in nephrotoxicity. J Am Soc Nephrol 2008;19:923-932.

7. Ramesh G, Reeves WB. TNF-alpha mediates chemokine and cytokine expression and renal injury in cisplatin nephrotoxicity. J Clin Invest 2002;110:835-842. 
8. Wang W, Brian RW, Ramesh G. Netrin-1 and kidney injury. I. Netrin-1 protects against ischemia-reperfusion injury of the kidney. Am J Physiol Renal Physiol 2008;294:F739-F747.

9. Ly NP, Komatsuzaki K, Fraser IP, et al. Netrin-1 inhibits leukocyte migration in vitro and in vivo. PNAS 2005;102:14729-14734.

10. Nguyen A, Cai $H$. Netrin-1 induces angiogenesis via a DCCdependent ERK1/2-eNOS feed-forward mechanism. PNAS 2006;103: 6530-6535.

11. Wilson BD, li M, Park KW, et al. Netrins promote developmental and therapeutic angiogenesis. Science 2006;313:640-644.

12. Navankasattusas S, Whitehead KJ, Suli A, et al. The netrin receptor UNC5B promotes angiogenesis in specific vascular beds. Development 2008;135:659-667.

13. Hebrok M, Reichardt LF. Brain meets pancreas: netrin, an axon guidance molecule, controls epithelial cell migration. Trends Cell Biol 2004;14:153-155.

14. Liu $Y$, Stein $E$, Oliver $T$, et al. Novel role for netrins in regulating epithelial behavior during lung branching morphogenesis. Curr Biol 2004;14:897-905.

15. Lu X, Le Noble F, Yuan L, et al. The netrin receptor UNC5B mediates guidance events controlling morphogenesis of the vascular system. Nature 2004:432:179-186.

16. Arakawa $\mathrm{H}$. Netrin-1 and its receptors in tumorigenesis. Nat Rev Cancer 2004;4:978-987.

17. Fitamant J, Guenebeaud C, Coissieux MM, et al. Netrin-1 expression confers a selective advantage for tumor cell survival in metastatic breast cancer. Proc Natl Acad Sci USA 2008;105:4850-4855.

18. Mazelin L, Bernet A, Bonod-Bidaud C, et al. Netrin-1 controls colorectal tumorigenesis by regulating apoptosis. Nature 2004 431:80-84.

19. Wang W, Reeves WB, Pays L, et al. Netrin-1 overexpression protects kidney from ischemia reperfusion injury by suppressing apoptosis. Am J Pathol 2009;175:1010-1018.

20. Ernest S, Bello-Reuss E. Expression and function of P-glycoprotein in a mouse kidney cell line. Am J Physiol 1995;269:C323-C333.

21. Wang W, Li C, Summer SN, et al. Role of AQP1 in endotoxemiainduced acute kidney injury. Am J Physiol Renal Physiol 2008;294 F1473-F1480.

22. Ramesh G, Zhang B, Uematsu S, et al. Endotoxin and cisplatin synergistically induce renal dysfunction and cytokine production in mice. Am J Physiol Renal Physiol 2007;293:F325-F332.

23. Ramesh G, Reeves WB. p38 MAP kinase inhibition ameliorates cisplatin nephrotoxicity in mice. Am J Physiol Renal Physiol 2005;289: F166-F174.
24. Bredesen DE, Mehlen $\mathrm{P}$, Rabizadeh $\mathrm{S}$. Apoptosis and dependence receptors: a molecular basis for cellular addiction. Physiol Rev 2004; 84:411-430.

25. Mehlen $\mathrm{P}$, Bredesen DE. The dependence receptor hypothesis. Apoptosis 2004;9:37-49.

26. Wei Q, Dong G, Yang T, et al. Activation and involvement of p53 in cisplatin-induced nephrotoxicity. Am J Physiol Renal Physiol 2007;293: F1282-F1291.

27. Reeves WB, Kwon O, Ramesh G. Netrin-1 and kidney injury. II. Netrin-1 is an early biomarker of acute kidney injury. Am J Physiol Renal Physiol 2008;294:F731-F738.

28. Fan $Y$, Shen $F$, Chen $Y$, et al. Overexpression of netrin-1 induces neovascularization in the adult mouse brain. J Cereb Blood Flow Metab 2008;28:1543-1551.

29. Llambi F, Causeret F, Bloch-Gallego E, et al. Netrin-1 acts as a survival factor via its receptors UNC5H and DCC. EMBO J 2001;20:2715-2722.

30. Wu TW, Li WW, Li H. Netrin-1 attenuates ischemic stroke-induced apoptosis. Neuroscience 2008;156:475-482.

31. Guenebeaud C, Goldschneider D, Castets $M$, et al. The dependence receptor UNC5H2/B triggers apoptosis via PP2A-mediated dephosphorylation of DAP kinase. Mol Cell 2010;40:863-876.

32. Forcet $C, Y e X, G r a n g e r ~ L$, et al. The dependence receptor DCC (deleted in colorectal cancer) defines an alternative mechanism for caspase activation. PNAS 2001;98:3416-3421.

33. Jiang $M$, Dong $Z$. Regulation and pathological role of $p 53$ in cisplatin nephrotoxicity. J Pharmacol Exp Ther 2008;327:300-307.

34. Tanikawa C, Matsuda K, Fukuda S, et al. p53RDL1 regulates p53 dependent apoptosis. Nat Cell Biol 2003;5:216-223.

35. Ramesh G, Kimball SR, Jefferson LS, et al. Endotoxin and cisplatin synergistically stimulate TNF-alpha production by renal epithelial cells. Am J Physiol Renal Physiol 2007;292:F812-F819.

36. Tadagavadi RK, Wang W, Ramesh G. Netrin-1 regulates Th1/Th2/Th17 cytokine production and inflammation through UNC5B receptor and protects kidney against ischemia-reperfusion injury. J Immunol 2010; 185:3750-3758.

37. Matsukawa A, Hogaboam CM, Lukacs NW, et al. Expression and contribution of endogenous IL-13 in an experimental model of sepsis. J Immunol 2000;164:2738-2744.

38. Manna SK, Aggarwal BB. IL-13 suppresses TNF-induced activation of nuclear factor-\{kappa\}B, activation protein-1, apoptosis. J Immunol 1998;161:2863-2872.

39. Sandovici $\mathrm{M}$, Henning $\mathrm{RH}$, van Goor $\mathrm{H}$, et al. Systemic gene therapy with interleukin-13 attenuates renal ischemia-reperfusion injury. Kidney Int 2008;73:1364-1373. 